\title{
Carcinoma de Meibômio como segundo tumor em portadores de retinobastoma: relato de dois casos
}

\author{
Eyelid tumor in anophthalmic socket due to retinoblastoma: report of two cases
}

Silvia Narikawa ${ }^{1}$, Mariângela Esther Alencar Marques², Silvana Artioli Schellini ${ }^{3}$

\section{RESUMO}

O retinoblastoma pode acometer um ou os dois olhos, ocorrendo de forma hereditária ou esporádica. O portador dessa doença pode desenvolver, a longo prazo, outros tipos de tumores não oculares. Relatamos dois pacientes: o filho, portador de cavidade anoftálmica bilateral após remoção dos olhos devido ao retinoblastoma, que apresentou segundo tumor palpebral unilateral, afetando a pálpebra superior; e o pai, portador de cavidade anoftálmica unilateral também por remoção de retinoblastoma e que apresentou tumoração de crescimento rápido na pálpebra superior ipsilateral ao olho enucleado. O exame imuno-histoquímico de ambas as lesões excisadas revelou que se tratava de carcinoma de glândulas sebáceas. Os pacientes evoluíram bem após a remoção do tumor palpebral e, até o momento, não apresentam sinais de recidiva ou metástase.

Descritores: Anoftalmia; Retinoblastoma; Adenocarcinoma sebáceo; Neoplasias palpebrais; Relato de casos; Humanos; Masculino; Adulto; Meia-idade

\begin{abstract}
Retinoblastoma is a unilateral or bilateral intraocular tumor, occurring as a hereditary or sporadic tumor. Another not ocular lesion is a possibility for retinoblastoma carriers. The authors report the cases of two patients: a son with bilateral anophthalmic socket due to retinoblastoma, who presented unilateral upper eyelid tumor; and his father with unilateral anophthalmic socket secondary to enucleation due to retinoblastoma and a tumor growing fast in the upper eyelid at the same side of the anophthalmic cavity. Lesions were diagnosed as sebaceous carcinoma confirmed by immunohistochemical exam. Both patients had the eyelid tumors removed, with good resolution. None of them have presented either signs of recurrence or metastasis.
\end{abstract}

Keywords: Anophthalmos; Retinoblastoma; Adenocarcinoma, sebaceous; Eyelid neoplasms; Case reports; Humans; Male; Adult; Middle aged

\section{INTRODUÇÃO}

O retinoblastoma é a neoplasia intraocular mais comum na infância. Ocorre de duas formas: esporádica ou hereditária, podendo afetar um ou ambos os olhos ${ }^{(1)}$. Cerca de dois terços de todos os casos são unilaterais e um terço, bilaterais(2).

Crianças portadoras de retinoblastoma apresentam risco para três condições que podem ameaçar a vida: metástase do retinoblastoma, tumor intracraniano (retinoblastoma trilateral) e tumores secundários não oculares ${ }^{(2)}$.

Indivíduos com retinoblastoma hereditário possuem aproximadamente $4 \%$ de chance de desenvolver um câncer secundário durante os primeiros 10 anos de seguimento, 18\% durante os primeiros 20 anos e $26 \%$ dentro de 30 anos $^{(3)}$.

Crianças com mutação germinal no gene RB1 que sobreviveram ao retinoblastoma hereditário, possuem alto risco de desenvolverem outros tumores primários e também outras neoplasias não oculares $^{(4)}$.

Os tumores secundários mais frequentes nesses casos são o osteossarcoma, hemangiopericitoma, condrossarcoma, rabdomiossarcoma, neuroblastoma, glioma, leucemia, carcinoma de glândula sebácea, carcinoma espinocelular e melanoma(2).

O retinoblastoma deve ser visto não apenas como um câncer intraocular, mas também como um protótipo de câncer hereditário em humanos ${ }^{(4)}$.
Este relato de caso está sendo feito por estarmos acompanhando dois portadores de cavidade anoftálmica após remoção do olho devido ao retinoblastoma e que apresentaram carcinoma sebáceo palpebral como segundo tumor. O fato mais sui generis destes dois casos é que se trata de pai e filho.

\section{RELATO DE CASOS}

\section{Caso 1}

RTK, masculino, 31 anos, descendente de japoneses, natural de Mirandópolis - SP. Portador de cavidade anoftálmica bilateral desde os três anos de idade, secundária a enucleação devido a retinoblastoma bilateral. Este é o único filho de mãe sadia e pai que teve retinoblastoma unilateral. Em outubro de 2007 foi atendido no serviço de Plástica Ocular da Faculdade de Medicina de Botucatu UNESP, com queixa de lesão na pálpebra superior do olho esquerdo, em crescimento há um ano. No exame o paciente apresentava extensa lesão elevada, acometendo toda a placa tarsal da pálpebra superior, de cor amarelada, sem sinais flogísticos e com cílios palpebrais normais, endurecida à palpação (Figura 1). Foi feita a hipótese diagnóstica de carcinoma sebáceo, diagnóstico confirmado por exame citológico por raspado da lesão tarsal, que foi positivo para neoplasia, sugestivo de carcinoma. O paciente foi então submetido à cirurgia para exérese do tumor com ampla margem de seguran-

Submitted for publication: January 26, 2011

Accepted for publication: August 1, 2011

Study carried out at the Faculdade de Medicina de Botucatu - UNESP.

${ }^{1}$ Physician, Ophthalmology Department, Otorrinolaringologia e Cabeça e Pescoço, Faculdade de Medicina, Universidade Estadual Paulista - UNESP - Botucatu (SP), Brazil.

2 Physician, Pathology Department, Faculdade de Medicina de Botucatu - UNESP - Botucatu (SP), Brazil.

${ }_{3}$ Prazil.

Medicina, Universidade Estadual Paulista - UNESP - Botucatu (SP), Brazil.

Funding: No specific financial support was available for this study.

Disclosure of potential conflicts of interest: S.Narikawa, None; M.E.A.Marques, None; S.A.Schellini, None.

Correspondence address: Silvana Artioli Schellini. Depto de OFT/ORL/CCP - Faculdade de Medicina de Botucatu - UNESP - Campus Universitário Botucatu - (SP) - 18609-970 - Brazil

E-mail: sartioli@fmb.unesp.br 
ça, seguida de reconstrução total da pálpebra superior do olho esquerdo usando retalhos e enxertos. O exame anatomopatológico da lesão excisada foi compatível com neoplasia maligna pouco diferenciada, de células epitelióides, com margens livres. O estudo imuno-histoquímico foi positivo para os anticorpos AE1/AE3, EMA, CD 15, Breast - 2 e p 63 (Figuras 2 e 4), definindo o diagnóstico de carcinoma de glândulas sebáceas.

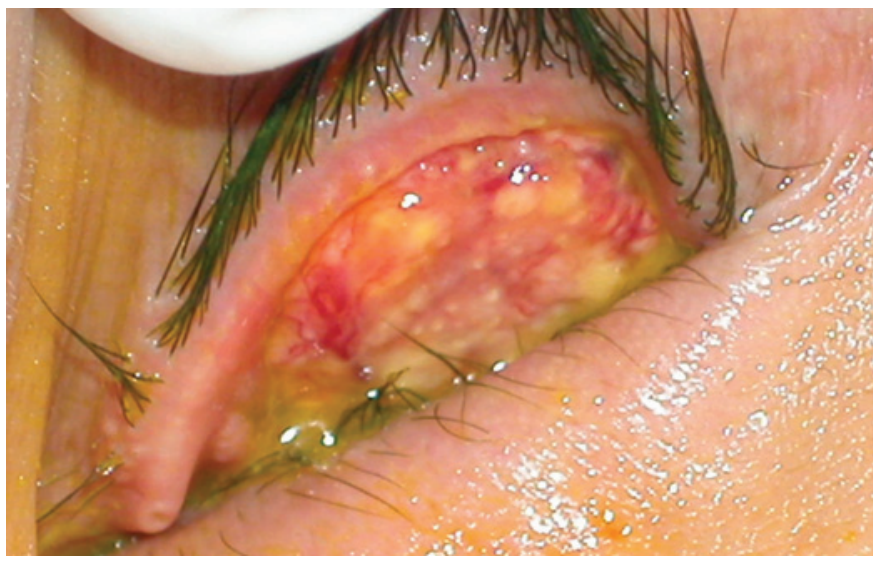

Figura 1. Aspecto da lesão palpebral do paciente RTK.

\section{CASO 2}

SK, masculino, descendente de japoneses, natural de Mirandópolis - SP, 63 anos, pai do paciente descrito no caso 1. Também portador de cavidade anoftálmica à esquerda, devido à remoção do olho por retinoblastoma. Em março de 2009 foi atendido com queixa de surgimento de nódulo na pálpebra superior esquerda há três meses. No exame, apresentava lesão endurecida, sem sinais flogísticos, localizada na placa tarsal da pálpebra superior esquerda. Foi feita a hipótese diagnóstica de carcinoma sebáceo e submetido à cirurgia para exérese do tumor (Figura 3, A e B).

Foi feita a exérese do tumor com reconstrução da pálpebra superior esquerda. O anatomopatológico da lesão excisada foi compatível com carcinoma sebáceo, com margens livres. O estudo imuno-histoquímico foi positivo para os anticorpos EMA, CEA, CD 15 e Breast - 2 (Figuras 2 e 4).

\section{DISCUSSÃO}

O retinoblastoma atualmente possui prognóstico bom, com a cura em cinco anos da maioria das crianças tratadas, desde que o diagnóstico seja precoce e o tratamento efetivo. Com a sobrevida a longo prazo, existe o risco de se desenvolver um segundo tumor não ocular ${ }^{(1)}$, risco esse, aumentado pelo uso da radioterapia(5).
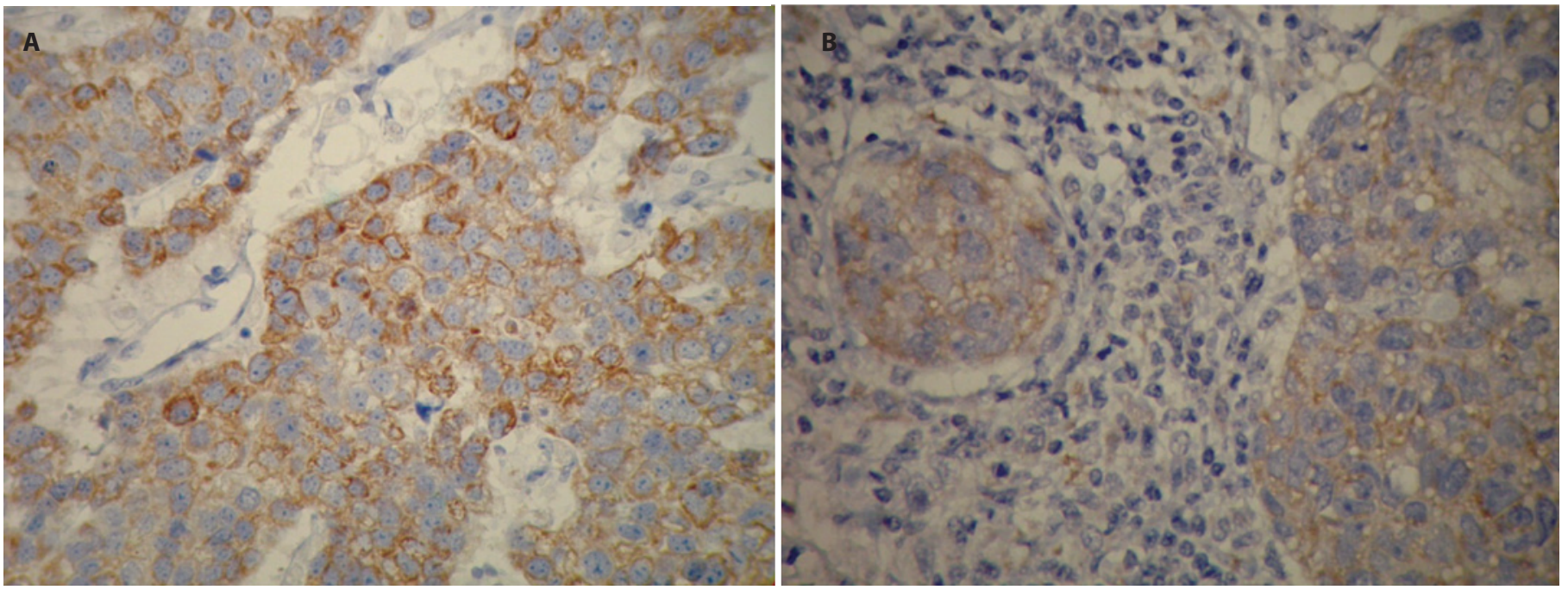

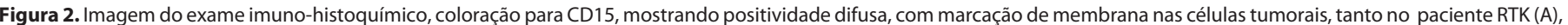
como no SK (B). AB 400X.
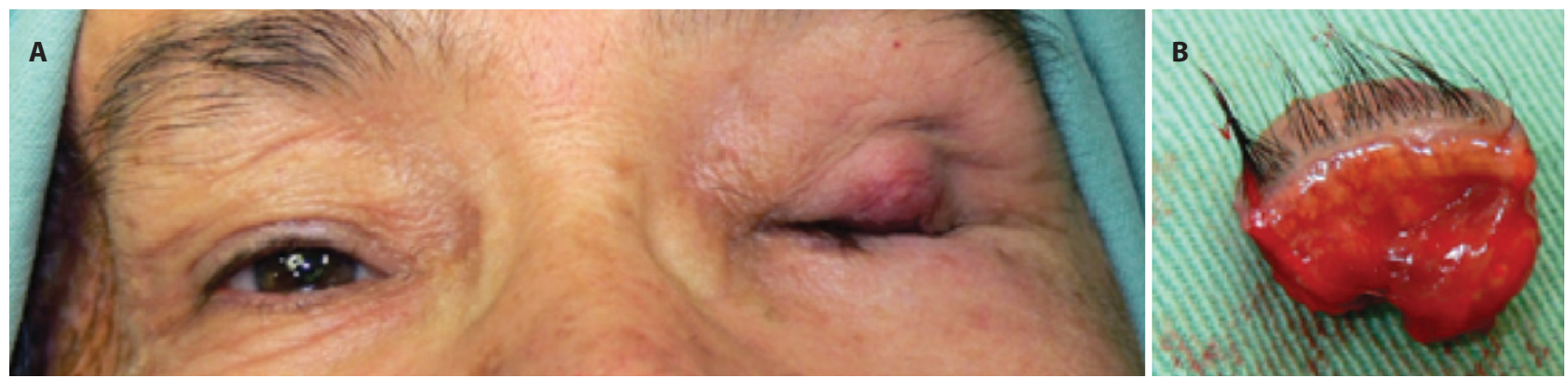

Figura 3. A) Aspecto da lesão palpebral do paciente SK. B) Lesão do paciente SK imediatamente após exérese. 

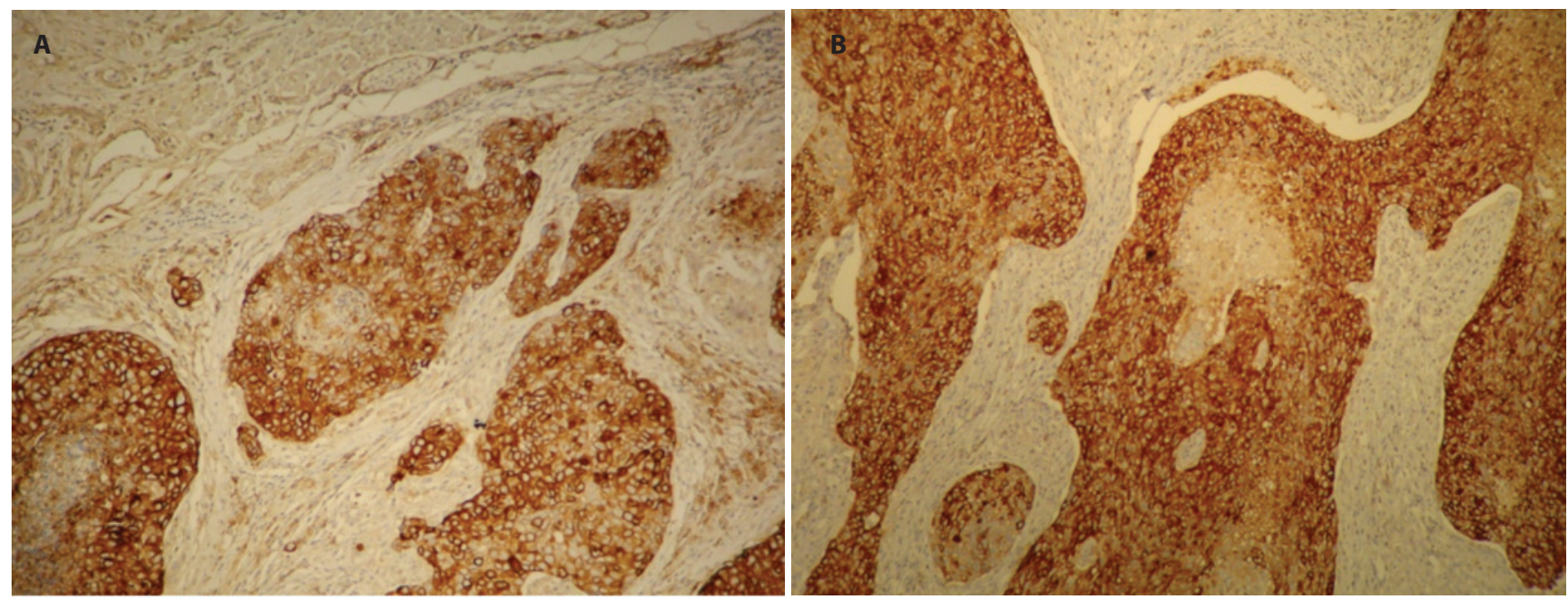

Figura 4. Antígeno de membrana epitelial (EMA) fortemente positivo nas células tunıorais. A) Paciente RTK, B) Paciente SK.ABC 200X.

Nos casos relatados, os portadores de cavidade anoftálmica secundária ao retinoblastoma apresentaram, em idades diferentes, um segundo tumor não ocular, provavelmente devido a sua associação com a mutação no gene RB1. O tumor apresentado por esses pacientes foi o carcinoma de glândulas sebáceas, localizado nas pálpebras superiores, ambos com apresentação unilateral.

O carcinoma de glândulas sebáceas é uma neoplasia maligna que apresenta agressividade local e pode evoluir com metástases para linfonodos e órgãos distantes. Ocorre principalmente na pálpebra superior e sua apresentação clínica mais comum é como um nódulo solitário, firme, indolor e fixo ao tarso ${ }^{(6)}$. A conjuntiva tarsal é acometida em $100 \%$ dos $\operatorname{casos}^{(7)}$.

O estudo imuno-histoquímico no carcinoma sebáceo é positivo para o antígeno de membrana epitelial $(E M A)^{(8)}$, o que ocorreu em ambos os casos.

Como a maioria dos tumores palpebrais são benignos e o carcinoma sebáceo pode mimetizar uma condição inflamatória local como um calázio ou blefarite, muitos pacientes sofrem com a demora no diagnóstico ${ }^{(7,8)}$, como ocorreu no caso 1 (RTK), que foi diagnosticado após um ano do surgimento da lesão, apesar de ter sido avaliado por vários especialistas, e nos chegou com lesão extensa, acometendo toda a pálpebra superior e até parte da inferior.

O prognóstico do carcinoma sebáceo é similar ao do carcinoma espinocelular da pele ${ }^{(9)}$. Felizmente, nos dois casos relatados, o diagnóstico foi feito em tempo de se remover com sucesso toda a lesão. Até o momento, ambos os pacientes não apresentaram sinais de recidiva ou metástases para linfonodos locais.

\section{CONCLUSÃO}

Os casos relatados reforçam a conhecida tendência de apresentação de segundo tumor em portadores de retinoblastoma. Ambos tiveram a remoção do tumor na infância, sendo o filho portador de lesão bilateral e o pai unilateral. Ambos tiveram carcinoma de células sebáceas na pálpebra, com evolução favorável até o presente momento.

\section{REFERÊNCIAS}

1. Schlienger P, Campana F, Vilcoq JR, Asselain B, Dendale R, Desjardins L, et al. Nonocular second primary tumors after retinoblastoma: retrospective study of 111 patients treated by electron beam radiotherapy with or without TEM. Am J Clin Oncol. 2004;27(4):411-9.

2. Shields $C L$, Shields JA. Basic understanding of current classification and management of retinoblastoma. Cur Opin Ophthalmol. 2006;17(3):228-34.

3. Roarty JD, McLean IW, Zimmerman LE. Incidence of second neoplasms in patients with bilateral retinoblastoma. Ophthalmology. 1998;9(11)5:1538-7.

4. Lyle CE, Rodriguez-Galindo C, Wilson MW. Tumores não oculares. In: Singh AD. Oncologia oftalmológica clínica. Rio de Janeiro: Cultura Médica; 2009. p. 209.

5. Wong FL, Boice JD, Abramson DH, Tarone RE, Kleinerman RA, Stovall M, et al. Cancer incidence after retinoblastoma. Radiation dose and sarcoma risk. JAMA. 1997;278(15): 12627. Comment in: JAMA. 1997;278(15):1284-5

6. Rosner M. Carcinoma glandular sebáceo. In: Singh AD. Oncologia oftalmológica clínica. Rio de Janeiro: Cultura Médica; 2009. p.45.

7. Shields JA, Demirci H, Marr BP, Eagle RC Jr, Stefanyszyn M, Shields CL. Conjunctival epithelial involvement by eyelid sebaceous carcinoma. The $2003 \mathrm{~J}$. Howard Stokes lecture. Ophthal Plast Reconstr Surg. 2005;21(2):92-6.

8. Deprez M, Uffer S. Clinicopathological features of eyelid skin tumors. A retrospective study of 5504 cases and review of literature. Am J Dermatopathol. 2009;31(3):256-62.

9. Schlauder SM, Morgan MB. Cutaneous neoplasms with sebaceous differentiation: a review. Pathology Case Reviews. 2007;12:79-85. 